\title{
Melting heat transfer in rectangular cavity filled with ice slurry heated from below
}

\author{
Taimei MIYAGAWA*, Takahiro OKABE*, Takuro MIYANISHI*, Takuma KOGAWA**, Hiroyuki MURATA* \\ and Koji FUMOTO*** \\ * Graduate School of Science and Technology, Hirosaki University \\ 3 Bunkyocho, Hirosaki, Aomori, 036-8561, Japan \\ Taimei MIYAGAWA and Takahiro OKABE contributed equally. \\ E-mail: miyagawa@hirosaki-u.ac.jp \\ ** National Institute of Technology, Hachinohe College \\ 16-1 Uwanotai, Tamonoki, Hachinohe, Aomori, 039-1192, Japan \\ *** Department of Mechanical Engineering, Aoyama Gakuin University \\ 5-10-1 Fuchinobe, Sagamihara, Kanagawa, 252-5258, Japan
}

Received: 25 March 2019; Revised: 27 May 2019; Accepted: 13 August 2019

\begin{abstract}
Ice slurry is a homogeneous mixture of small ice particles and a carrier liquid. It is widely used in many fields. Previous studies have gradually clarified its heat transfer in high ice packing factor (IPF). However, only a few studies have focused on the mechanism of heat transfer with low IPF in a cavity heated from below. The objective of this study is to experimentally clarify the melting heat transfer of ice slurry in a cavity heated from below with low IPF to develop a direct contact medical cooling system. To observe the melting behavior of ice slurry, the test section was made of acrylic resin $(100 \mathrm{~mm} \times 60 \mathrm{~mm} \times 30 \mathrm{~mm})$ and a silicone rubber heater that was used for heating under constant heat flux conditions. We measured the surface temperature of the heater and the liquid thickness. We showed that the melting process can be divided into three stages. In the first stage, heat conduction dominated the process of heat transfer and the temperature of the heater rapidly increased. In the second stage, natural convection heat transfer dominated the process of heat transfer that increased the melting rate of ice slurry and decreased the temperature of the heater. In the third stage, heat conduction dominated the process of heat transfer in the concentration stratification. This led to a decrease in the melting rate and an increase in the temperature of the heater. Our result also showed that the melting process of the ice slurry is slow enough to consider it the quasi-steady state in the range of $10^{4}<R a^{*}<10^{7}$ as compared to the development of the velocity and temperature fields.
\end{abstract}

Keywords : Ice slurry, Melting heat transfer, Natural convection, Melting behavior, Image processing

\section{Introduction}

Ice slurry is a homogeneous mixture of small ice particles $(0.1-0.6 \mathrm{~mm})$ and a carrier liquid. It has a high energy storage density and fast cooling rate because of the latent heat and large heat transfer surface area created by numerous ice particles (Kauffeld et al., 2010). Therefore, it is used in many fields such as heat storage system, fishery (Piñeiro et al., 2004), and medical cooling (Orvieto et al., 2007). Sodium chloride, ethanol, ethylene glycol, and propylene glycol are used to depress the freezing point of water. We developed the method to produce ice slurry using saline (Fumoto et al., 2013). In this study, we used sodium chloride solution as the carrier liquid to develop an application for medical cooling.

Ice slurry is expected to be applied to kidney cooling during laparoscopic surgery (Kauffeld et al., 2010). It is important to understand the basic mechanisms of the melting heat transfer to develop the efficient ice slurry based medical cooling system. Recently, the ice slurry in pipe flow has been actively researched (Kumano et al., 2010 and Knodel et al., 2010). However, it is difficult to efficiently cool the organ by using this system during laparoscopic surgery. On the other 
hand, direct contact cooling is suitable for efficient cooling of the organ in a limited space such as the abdominal cavity because of adhesion of ice slurry.

Several studies have been previously conducted that focus on direct contact cooling using ice slurry. Fukusako et al. (1992, 1993) investigated the melting heat transfer of ice slurry in a rectangular cavity with a heated vertical wall or bottom wall when ice packing factor (IPF), which represents the mass fraction of ice phase, is $65 \%$. Their experiment indicated that the ice slurry phase moves above the liquid phase owing to buoyancy and the liquid phase becomes larger as the ice slurry melts. In the early stage of melting, heat conduction becomes dominant and natural convection occurs in the liquid phase. Concentration stratification was formed as the liquid thickened and its length changed with heat flux and the initial concentration of an aqueous solution. They also indicated that the formation and growth of double-diffusive layer significantly affects the melting process of ice slurry.

Kousksou et al. (2010) numerically investigated the melting behavior of ice slurry heated from below. Their research showed that natural convection appears when Rayleigh number of liquid phase exceeds a threshold value of 1708 and the pattern of convection is similar to that of classical Rayleigh-Bénard convection.

Ice in a cavity heated from below has been studied since the 1960s. Fukusako et al. (1977) reviewed the melting heat transfer in a rectangular cavity filled with ice heated from below. Yen (1968) and Kim et al. (2008) suggested that the onset of natural convection in liquid phase is affected by the temperature difference between the heater and the melting surface. Fukusako et al. (1977) reorganized Yen's data and showed that the critical Rayleigh number increases with increasing temperature of the heater.

Lately, the melting heat transfer of phase change material (PCM) in a cavity has actively been studied. Ice slurry is a type of PCM. Joneidi et al. (2017) experimentally investigated the effects of heat flux and inclination angle on the melting heat transfer. Madruga and Curbelo (2018) numerically investigated the melting of PCM and found that the Nusselt number scaled with the Rayleigh number in turbulent regime.

The main difference between ice and ice slurry is the solvent. Usually, sodium chloride and ethanol, among others, are used to depress the freezing point of water. The solvent concentration affects the melting heat transfer in the cavity (Fukusako et al., 1993). This problem is called double-diffusive convection and the flow and temperature fields become complex.

Previous studies have gradually clarified the heat transfer of PCM and ice slurry with high IPF. However, few studies have focused on the mechanism of heat transfer of ice slurry with low IPF in a cavity heated from below. The advantage of the ice slurry with low IPF is its transportability in the narrow tube such as the catheter. Ice slurry with low IPF is expected to be applied to organ cooling in laparoscopic surgery.

The objective of this study is to experimentally clarify the melting heat transfer of ice slurry in a cavity heated from below with low IPF to develop a direct contact medical cooling system. In this paper, we investigated the effects of IPF and heat flux on the surface temperature of heater, melting rate, and melting heat transfer of ice slurry.

\section{Method}

\subsection{Experimental setup}

Figure 1 shows a schematic diagram of the experimental setup used in this study. This system consists of the test section, digital multimeter, data logger, personal computer (PC), and direct current (DC) supply. To observe the melting behavior of ice slurry, the test section was made of acrylic resin $(100 \mathrm{~mm} \times 60 \mathrm{~mm} \times 30 \mathrm{~mm})$ as shown in Fig.1 (b). A silicone rubber heater was used for heating under the constant heat flux conditions. Left, right, top, and back walls were insulated and the shape and position of melting surface were observed by a digital camera (Nikon D500) placed in front of the test section. A copper plate with thickness of $0.3 \mathrm{~mm}$ was put on the heater for the uniform distribution of temperature. Three thermistors were used to measure the surface temperature of the heater. To prevent liquid leakage, a plastic wrap with thickness of $10 \mu \mathrm{m}$ was applied to the bottom surface of the acrylic case. Four thermocouples were installed to monitor the heat loss of the heater.

\subsection{Experimental procedures}

To reduce the heat loss from the test section, the acrylic case was cooled down to $-5{ }^{\circ} \mathrm{C}$ by using refrigerator before the experiment. Then, ice slurry was put into the acrylic case. The base fluid of ice slurry was $3.0 \mathrm{wt} \%$ of the sodium chloride aqueous solution. Ice slurry with different $\operatorname{IPF}(15,20$, and 30\%) was used for this study. A constant heat flux $\left(q=2500\right.$ and $\left.3600 \mathrm{~W} / \mathrm{m}^{2}\right)$ was applied to the acrylic case from below. The melting behavior of ice slurry was recorded with a camera. We measured the surface temperature of the heater and the liquid thickness for each condition. 
(a)

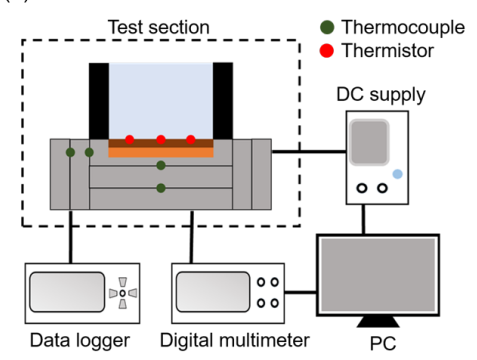

(b)

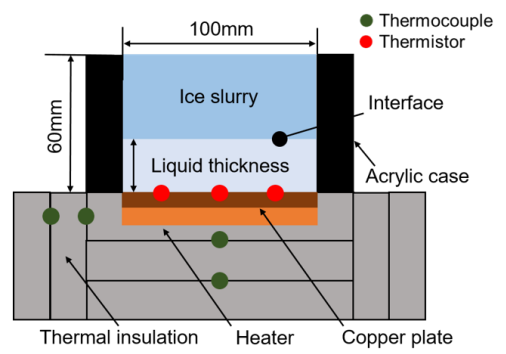

Fig. 1 Experimental setup used in this study: (a) Overall view of experimental setup and (b) details of test section.

To evaluate the liquid thickness quantitatively, image processing with MATLAB (MathWorks, Inc) was implemented. Figure 2 shows the procedure of image processing developed in this study. Using the original images (Fig.2 (a)), binary images (Fig.2 (b)) were generated by the following procedure. First, original images were converted to grayscale. Then, when the brightness value of each pixel exceeded the threshold, the pixel was replaced with white pixel; otherwise, it was replaced with black pixel. The threshold was defined such that the melting surface in the binary image and it in the original image were matched. In this procedure, the ice slurry phase was expressed as white pixels. The average thickness of the ice slurry was calculated by dividing the area of ice slurry phase by width of the cavity at each instance of time. The liquid thickness $(L)$ was obtained by subtracting the ice slurry thickness from the height of acrylic case. For each experimental condition, a set of images were taken during the experiment. In this study, we collected data until the liquid thickness reached $50 \mathrm{~mm}$. We confirmed from the side view of the test section that the melting surface is almost flat in the depth direction while the ice slurry is melting.

To evaluate the melting heat transfer in the liquid phase, we calculated the following parameters. First, heat transfer coefficient $(h)\left[\mathrm{W} /\left(\mathrm{m}^{2} \mathrm{~K}\right)\right]$ was calculated by

$$
h=\frac{q}{T-T_{b}}
$$

where $q$ is heat flux $\left[\mathrm{W} / \mathrm{m}^{2}\right.$ ] calculated from the input power of the heater, $T$ is average temperature of the heater [ ${ }^{\circ} \mathrm{C}$, and $T_{b}$ is the temperature of bulk fluid $\left[{ }^{\circ} \mathrm{C}\right]$. In this study, we assumed that the temperature in the liquid phase linearly increases from $T$ to $T_{m}$ (temperature of melting surface; $T_{m}=0{ }^{\circ} \mathrm{C}$ ). Thus, we defined $T_{b}=\left(T_{m}+T\right) / 2=T / 2$.

Second, modified Rayleigh number $\left(R a^{*}\right)$ [-] was calculated by

$$
R a^{*}=\frac{g \beta q L^{4}}{\alpha \lambda v}
$$

where $\alpha$ is thermal diffusivity $\left[\mathrm{m}^{2} / \mathrm{s}\right], \beta$ is the coefficient of thermal expansion $[1 / \mathrm{K}], \lambda$ is thermal conductivity $[\mathrm{W} /(\mathrm{m} \cdot \mathrm{K})]$, $v$ is kinematic viscosity $\left[\mathrm{m}^{2} / \mathrm{s}\right]$, and $g$ is the gravitational acceleration $\left[\mathrm{m} / \mathrm{s}^{2}\right]$. Note that thermophysical properties of the liquid phase at $T_{b}$ were used. Finally, Nusselt number $(\mathrm{Nu})$ [-] was calculated by

$$
N u=\frac{h L}{\lambda} .
$$

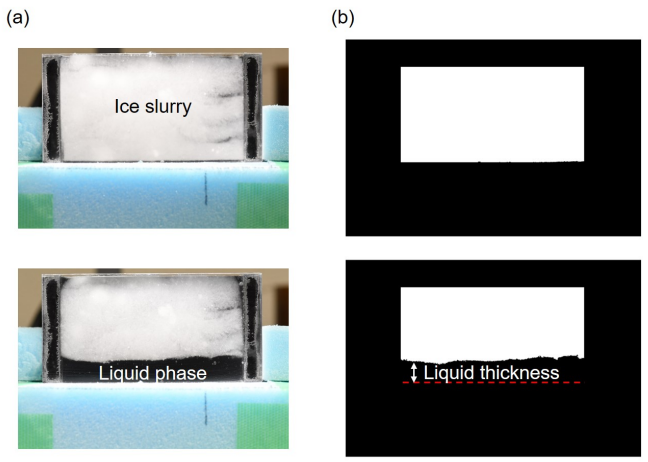

Fig. 2 Procedures of image processing for evaluation of liquid thickness: (a) Original images and (b) binary images. 


\section{Results and Discussion}

\subsection{Effect of IPF on temperature and melting behavior}

Figure 3 shows the time history of the average surface temperature of the heater $(T)$ and the average liquid thickness (L) for $q=2500$ and $3600 \mathrm{~W} / \mathrm{m}^{2}$ with different IPFs. The surface temperature of the heater rapidly increased on heating. In the early stage of melting, the buoyancy forces of melted ice slurry could not overcome the resistance imposed by viscous forces that caused the heat conduction to dominate the process of heat transfer in the liquid phase. This led to a significant increase in the surface temperature.

Then, the temperature gradually decreased with fluctuations owing to the Rayleigh-Bénard convection and the heat transfer coefficient increased in the liquid phase (Okabe et al., 2018). The melting rate of ice slurry increased owing to the increase in heat transfer from the heater. The local maximum temperature became higher in high IPF because the latent heat delayed the onset of the Rayleigh-Bénard convection. The thickness of the liquid phase during the Rayleigh-Bénard convection tends to decrease as IPF increases and increase as heat flux increases.

Fukusako et al. (1993) reported that a stable concentration stratification develops with an increasing thickness of the liquid phase. In our study, the concentration stratification seems to develop near the melting surface as with the study of Fukusako et al (1993). In the concentration stratification, heat conduction dominated the process of heat transfer. The Rayleigh-Bénard convection continued in the liquid phase. However, heat supply to the melting surface decreased owing to concentration stratification. Thus, the melting rate significantly decreased and the temperature again increased.

In this system, the melting process can be divided into three stages. In the first stage, heat conduction dominated the process of heat transfer, and the temperature of the heater rapidly increased. Then, buoyancy forces of the melted ice slurry overcame the resistance imposed by viscous forces and caused the Rayleigh-Beńard convection. In the second stage, natural convection heat transfer dominated the process of heat transfer and the melting rate of ice slurry increased and the temperature of the heater decreased. As the thickness of the liquid phase increased, the concentration stratification gradually developed. In the third stage, the heat conduction dominated the process of heat transfer in the concentration stratification. As a result, the heat supply to the melting surface decreased. This led to a decrease in the melting rate and an increases in the temperature of the heater. It takes a long time to melt because the amount of ice particles increases in the case of high IPF. Our results suggest that the ice slurry with high IPF can cool the heater for a long time, however, IPF must be chosen carefully owing to the rise of the temperature in the first stage.

Figure 4 shows the relationship between total input energy per unit area $(q \cdot t)$ and liquid thickness. For the same IPF, the thickness of liquid can be expressed as a function of $q \times t$ except for IPF $=30 \%$. This means that if the amount of input heat is the same, the amount of melting would also be the same, independent of the heat flux. However, the difference in the amount of melting between $q=2500$ and $3600 \mathrm{~W} / \mathrm{m}^{2}$ increases as IPF increases.

(a)

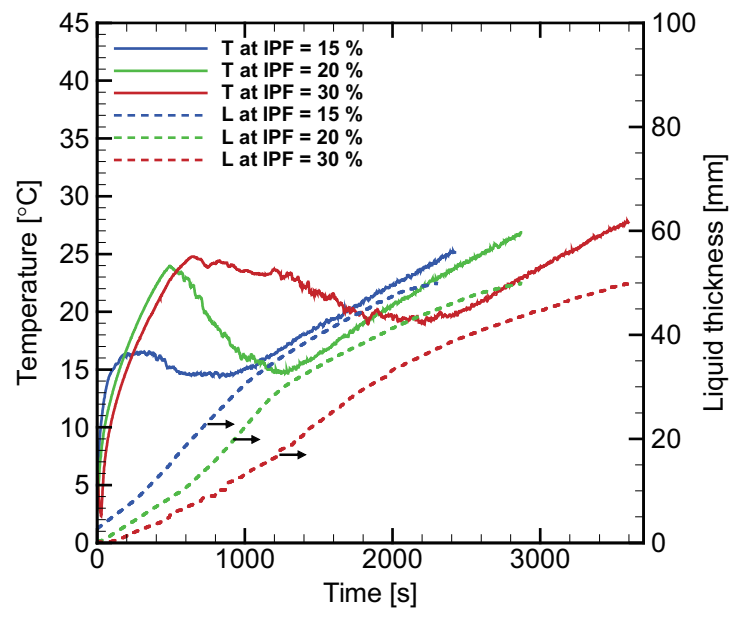

(b)

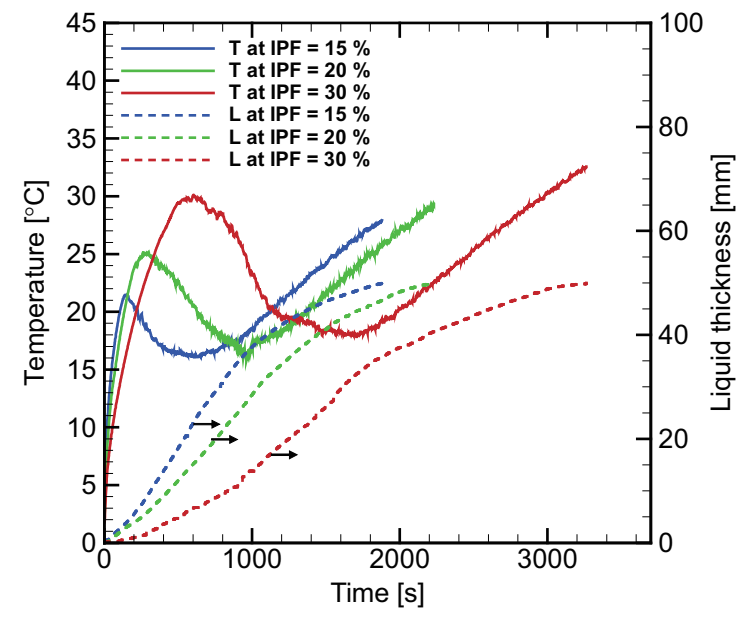

Fig. 3 Time history of surface temperature of heater and liquid thickness for (a) $q=2500 \mathrm{~W} / \mathrm{m}^{2}$ and (b) $q=3600$ $\mathrm{W} / \mathrm{m}^{2}$. The surface temperature of the heater is plotted as a solid line and the thickness of a liquid phase is plotted as a dashed line. Red, green, and blue lines represent the results of IPF $=15,20$, and $30 \%$, respectively. 


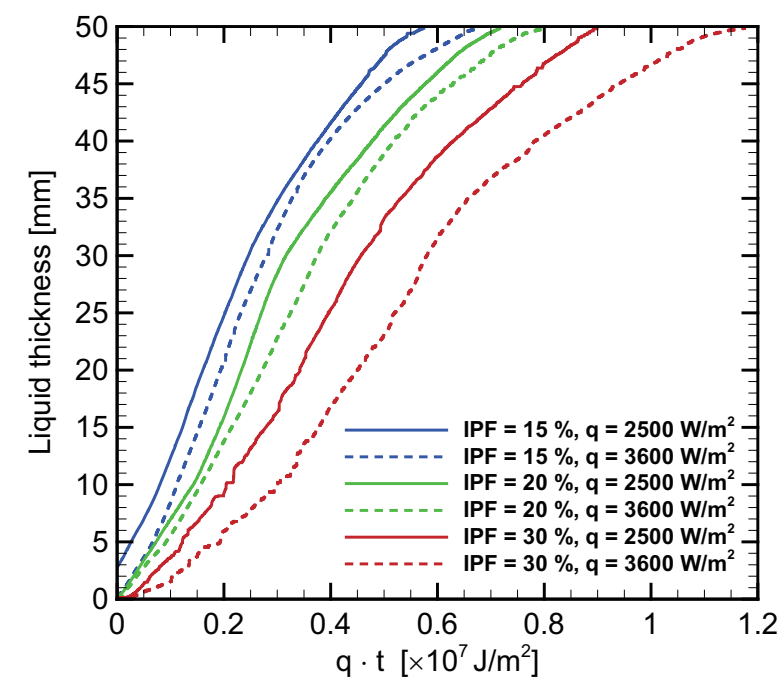

Fig. 4 Relationship between total input energy per unit area and liquid thickness. The results of $q=2500 \mathrm{~W} / \mathrm{m}^{2}$ are plotted as solid lines and those of $q=3600 \mathrm{~W} / \mathrm{m}^{2}$ are plotted as dashed lines. Red, green, and blue lines represent the result of IPF $=15,20$, and $30 \%$, respectively.

\subsection{Melting heat transfer of ice slurry}

Figure 5 shows the time history of the modified Rayleigh number. The modified Rayleigh number increases with time and the maximum modified Rayleigh number is almost the same under all conditions. The critical Rayleigh number, which is defined as the Rayleigh number when natural convection occurs in the liquid phase (black circles in Fig.5), tends to decrease with increasing heat flux when IPF is the same. Yen (1968) and Kim et al. (2008) showed that the critical Rayleigh number increased as the temperature difference between bulk fluid and heater increased when the ice in the cavity was heated from below. In this study, the temperature difference between heater and bulk fluid increased when the heat flux increased but the critical Rayleigh number decreased. This may be due to the different boundary conditions and the effect of the concentration field of sodium chloride.

Figure 6 shows the time history of the Nusselt number. Black circles represent the time when convection occurs in each IPF. The Nusselt number increased with time and reaches the maximum value when the surface temperature of the heater reaches the local maximum value (Fig.3). The increasing rate of the Nusselt number becomes large after the onset of the convection. The Nusselt number gradually decreases after the development of concentration stratification.

Figure 7 shows the relationship between the modified Rayleigh number and Nusselt number in the liquid phase for $q$ $=2500$ and $3600 \mathrm{~W} / \mathrm{m}^{2}$, and IPF $=15,20$, and $30 \%$. The relationship is almost consistent for all IPFs and heat flux. This result indicates that heat transfer in the melted ice slurry does not depend on IPF and heat flux.

We compared our results with the correlation equation for natural convection heat transfer of water in a horizontal layer (Hollands et al., 1975). When the modified Rayleigh number is less than $10^{4}$, the Nusselt number tends to become large as compared to the correlation equation owing to the effect of latent heat. However, our results were qualitatively similar to the correlation equation when the modified Rayleigh number was larger than $10^{4}$. When the concentration stratification developed in $R a>10^{7}$, the difference between our results and the correlation equation became large. This difference occurred owing to the definition of $L$ in Equations (2) and (3). Concentration stratification and Rayleigh-Bénard convection appeared on the stable and unstable layers of liquid thickness, respectively. Hence, we should use the length of the unstable layer instead of the liquid thickness as the definition of $L$ for accurate measurement of the Nusselt number and modified Rayleigh number.

These results indicated that the melting process of ice slurry is slow enough to regard it is quasi-steady state in the range of $10^{4}<R a^{*}<10^{7}$ compared with the development of the velocity and temperature field. Therefore, latent heat did not affect the natural convection heat transfer in the melted ice slurry and the mechanism of heat transfer was similar to water in this range.

We showed that the melting process can be divided into three stages. In the fast stage $\left(R a^{*}<10^{4}\right)$, the Nusselt number tends to become large as compared to the correlation equation owing to the effect of latent heat. In the second stage $\left(10^{4}<R a^{*}<10^{7}\right)$, natural convection heat transfer dominated the process of heat transfer, and the relationship 
between the Nusselt number and the modified Rayleigh number corresponds to it in the case of water. In the third stage $\left(R a^{*}>10^{7}\right)$, heat conduction dominated the process of heat transfer in the concentration stratification, and the Nusselt number is almost constant.

Some limitations exist in this study. First, we do not observe the flow pattern in the liquid phase. In this study, we measured the temperature of the heater, the liquid thickness, $\mathrm{Ra}$ and $\mathrm{Nu}$, and showed the effect of IPF and heat flux on them. To clarify the relationship among them in detail, the observation of the flow pattern in the liquid phase is need. Second, we assumed that $T_{b}=T / 2$. For accurate measurement of the Nusselt number, we must measure the temperature of the bulk fluid. Third, the temperature of the three thermistors was different up to $5{ }^{\circ} \mathrm{C}$ owing to the pattern of convection. We also need more thermistors to represent the temperature on the heater.

(a)

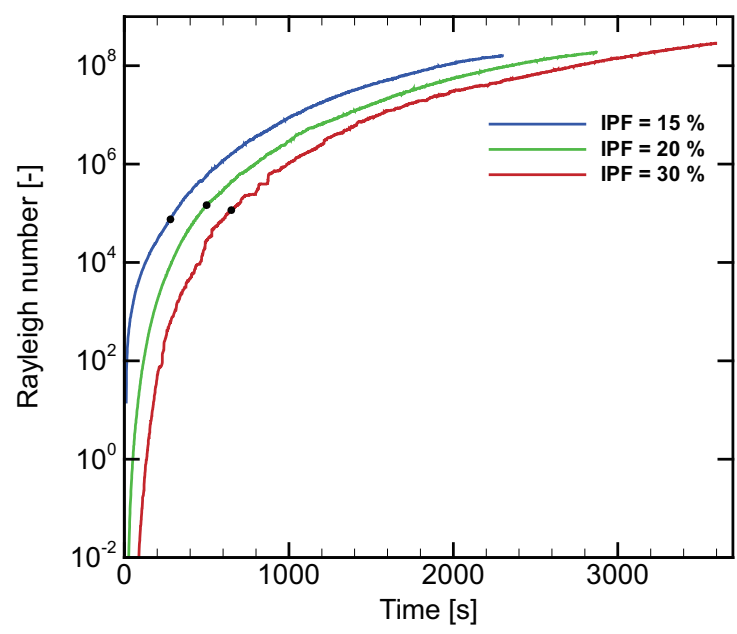

(b)

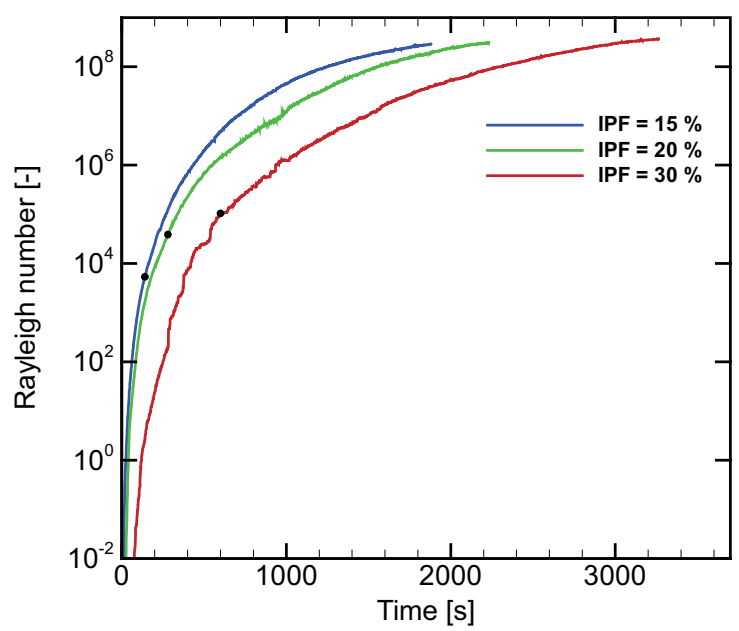

Fig. 5 Time history of modified Rayleigh number for (a) $q=2500 \mathrm{~W} / \mathrm{m}^{2}$ and (b) $q=3600 \mathrm{~W} / \mathrm{m}^{2}$. Blue, green, and red lines represent IPF $=15,20$, and $30 \%$, respectively. Black circles represent the time when convection occurs in each IPF.

(a)

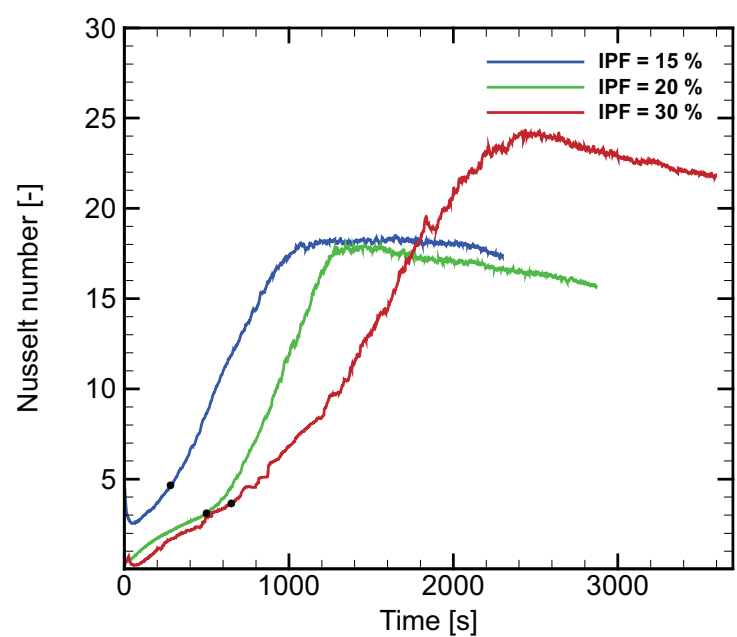

(b)

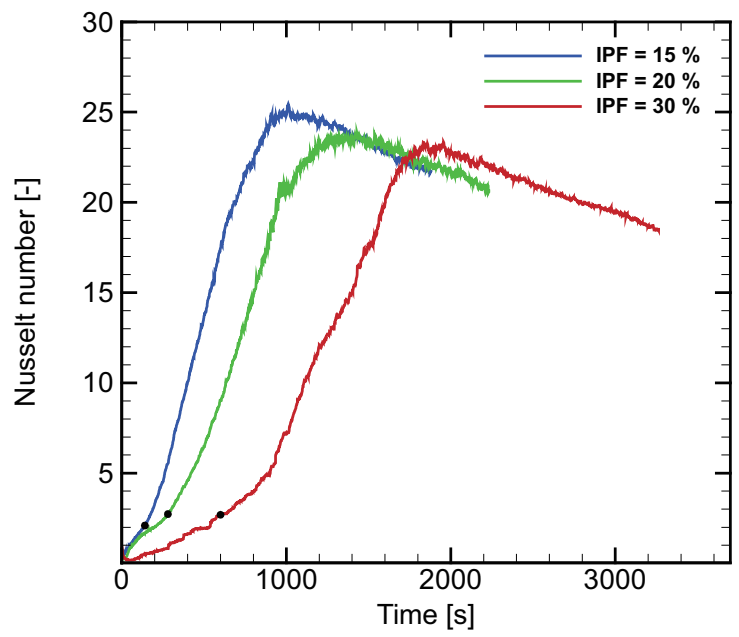

Fig. 6 Time history of Nusselt number for (a) $q=2500 \mathrm{~W} / \mathrm{m}^{2}$ and (b) $q=3600 \mathrm{~W} / \mathrm{m}^{2}$. Blue, green, and red lines represent IPF $=15,20$, and $30 \%$, respectively. Black circles represent the time when convection occurs in each IPF. 


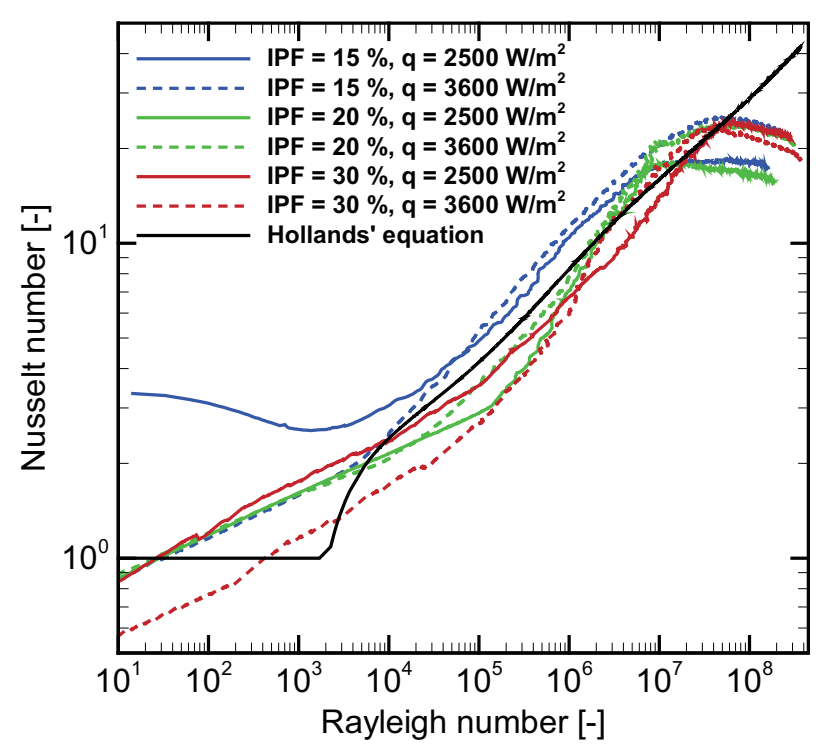

Fig. 7 Relationship between modified Rayleigh number and Nusselt number. Blue, green, and red lines represent $\mathrm{IPF}=15,20$, and $30 \%$, respectively. Solid and dashed lines represent $q=2500$ and $3600 \mathrm{~W} / \mathrm{m}^{2}$, respectively. Black line shows the correlation equation for water as stated by Hollands et al. (1975).

\section{Conclusion}

In this study, we observed the melting heat transfer of ice slurry in a cavity heated from below. We showed that the melting process can be divided into three stages. In the first stage, heat conduction dominated the process of heat transfer and the temperature of the heater rapidly increased. In the second stage, natural convection heat transfer dominated the process of the heat transfer, resulting in increased melting rate of ice slurry and decreased temperature of the heater. In the third stage, heat conduction dominated the process of heat transfer in the concentration stratification. This led to a decrease in the melting rate and an increase in the temperature of the heater.

We compared our results with the correlation equation for natural convection heat transfer of water in a horizontal layer. The relationship between the modified Rayleigh number and Nusselt number was found to be almost consistent for all IPFs and heat flux. Our result also indicated that the melting process of the ice slurry is slow enough to consider it quasi-steady state in the range of $10^{4}<R a<10^{7}$ as compared to the development of velocity and temperature fields.

\section{Acknowledgment}

This work was supported by Hirosaki University Guroukaru Fund.

\section{References}

Fukusako, S., Sugawara, M. and Seki, N., Onset of free convection and free convective heat transfer in a horizontal melt layer of ice, Journal of the Society of Mechanical Engineers, Vol.80, (1977), pp.445-450 (in Japanese).

Fukusako, S., Yamada, M. and Kim, M., Melting heat transfer of liquid ice in a rectangular cavity with heated vertical wall, Transactions of the Japan Society of Mechanical Engineers Series B, Vol.58, (1992), pp.2535-2542 (in Japanese).

Fukusako, S., Yamada, M. and Kim, M., Melting heat transfer of liquid ice in a rectangular cavity with heated bottom wall, Transactions of the Japan Society of Refrigerating and Air Conditioning Engineers, Vol.10, (1993), pp.273-281 (in Japanese).

Fumoto, K., Kawanami, T. and Inamura, T., Study on new ice slurry generator using $\mathrm{NaCl}$ aqueous solution at low concentration, International Journal of Air-Conditioning and Refrigeration, Vol.21, (2013), pp.1350004.

Hollands, K. G. T., Raithby, G. D. and Konicek, L., Correlation equations for free convection heat transfer in horizontal layers of air and water, International Journal of Heat and Mass Transfer, Vol.18, (1975), pp.879-884. 
Joneidi, M. H., Hosseini, M. J., Ranjbar, A. A. and Bahrampoury, R., Experimental investigation of phase change in a cavity for varying heat flux and inclination angles, Experimental Thermal and Fluid Science, Vol.88, (2017), pp.594607.

Kauffeld, M., Wang, M. J., Goldstein, V. and Kasza, K. E., Ice slurry applications, International Journal of Refrigeration, Vol.33, (2010), pp.1491-1505.

Kim, M. C., Choi, C. K. and Yoon D.-Y., Analysis of the onset of buoyancy-driven convection in a water layer formed by ice melting from below, International Journal of Heat and Mass Transfer, Vol.51, (2008), pp.5097-5101.

Knodel, B. D., France, D. M., Choi, U. S. and Wambsganss, M. W., Heat transfer and pressure drop in ice-water slurries, Applied Thermal Engineering, Vol.20, (2010), pp.671-685.

Kousksou, T., Arid, A., Majid, J. and Zeraouli, Y., Numerical modeling of double-diffusive convection in ice slurry storage tank, International Journal of Refrigeration, Vol.33, (2010), pp.1550-1518.

Kumano, H., Hirata, T., Shoji, R. and Shirakawa, M., Experimental study on heat transfer characteristics of ice slurry, International Journal of Refrigeration, Vol.33, (2010), pp.1540-1549.

Madruga, S. and Curbelo, J., Dynamic of plumes and scaling during the melting of a phase change material heated from below, International Journal of Heat and Mass Transfer, Vol.126, (2018), pp.206-220.

Okabe, T., Takada, S., Shirota, M., Inamura, T. and Fumoto, K., Fundamental study of heat transfer behavior of ice slurry on biological phantom with equivalent perfusion, Proceedings of 9th Asian Conference on Refrigeration and Air-conditioning (ACRA2018), (2018), ACRA2018-D231.

Orvieto, M. A., Zorn, K. C., Lyon, M. B., Tohurst, S. R., Rapp, D. E., Mikhail A. A., Oras, J. J., Son, H., Kasza, K. E., Brendler C. B. and Shalhav, A. L., Laparoscopic ice slurry coolant for renal hypothermia, Journal of Urology, Vol.177, (2007), pp.382-385.

Piñeiro, C., Barros-Velázquez, J. and Aubourg, S. P., Effects of newer slurry ice systems on the quality of aquatic food products: a comparative review versus flake-ice chilling methods, Trends in Food Science and Technology, Vol.15, (2004), pp.575-582.

Yen, Y.-C., Onset of convection in a layer of water formed by melting ice from below, Physics of Fluids, Vol.11, (1968), pp.1263-1270. 